\title{
AUTOSTEM PARA A INCLUSÃO: ADAPTACÃO DE UM GUIÃO PEDAGÓGICO PARA CRIANÇAS CEGAS OU COM BAIXA VISÃOO
}

\author{
Conceição Costa \\ Escola Superior de Educação de Coimbra \\ ccosta@esec.pt \\ Rosário Gomes
}

Núcleo de Integração e Aconselhamento - Serviços de Ação Social e Escolar - UC

rosario.gomes@sas.uc.pt

Luís Barata

Núcleo de Integração e Aconselhamento - Serviços de Açao Social e Escolar - UC

Graça Bidarra

Faculdade de Psicologia e de Ciências da Educação - UC

gbidarra@fpce.uc.pt

Valentim Alferes

Faculdade de Psicologia e de Ciências da Educação - UC

valferes@fpce.uc.pt

Anália Santos

Faculdade de Psicologia e de Ciências da Educação - UC

analigonc@gmail.com

Piedade Vaz-Rebelo

Faculdade de Psicologia e de Ciências da Educação da Universidade de Coimbra pvaz@fpce.uc.pt

Recepción Artículo: 28 mayo 2021

Admisión Evaluación: 28 mayo 2021

Informe Evaluador 1: 29 mayo 2021

Informe Evaluador 2: 02 junio 2021

Aprobación Publicación: 03 junio 2021

\section{RESUMO}

No sentido de promover a inclusão educativa nas atividades do projeto Erasmus + Automata for STEM nr 2018-1-PT01-KA201-047499, desenvolveu-se a adaptação para crianças cegas ou com baixa visão do guião pedagógico de um dos autómatos do projeto, visando, por um lado, promover a representação mental do protótipo e das partes que o constituem e, por outro, a construção do mesmo pelas crianças. Trata-se de um pássaro, feito com papel e cartão, cujo movimento provocado, através do movimento deslizar, é o bater das asas. Tendo em consideração a importância que a informação auditiva e 0 experienciar táctil assumem na cegueira e baixa visão, ao longo do processo de adaptação do guião foram desenvolvidos recursos adicionais, tais como folhas em relevo para uma exploração táctil das formas geométricas do brinquedo e ainda diversas descrições e instruções que o/a professor/a ou educador/a pode seguir para fomentar por exemplo a representação mental de conceitos geométricos pelas crianças. 


\title{
AUTOSTEM PARA A INCLUSÃO: ADAPTAÇÃO DE UM GUIÃO PEDAGÓGICO PARA CRIANÇAS CEGAS OU COM BAIXA VISÃO
}

Palavras-clave: autómato; STEM; imagens mentais; geometria; cegueira e baixa visão; educação inclusiva

\begin{abstract}
AutoSTEM for inclusion: adapting a teaching guide for blind and visual impaired children. In order to promote educational inclusion in the activities of the Erasmus+ Project Automata for STEM nr 2018-1PT01-KA201-047499, it was developed the adaptation for blind or visual impaired children of the pedagogical guide of one of the autómata of that project. The adaptation process aimed, on the one hand, to promote mental representation of the automaton and its parts, but also the construction of it by the children. It is a bird, made of paper and cardboard, whose movement is the beating of the wings, through sliding motion. Taking into account the importance that auditory information and the tactile experience assume in blindness and viusal impaired people, throughout the adaptation process, additional resources were developed, namely embossed sheets for a tactile exploration of the geometric shapes of the toy, and also several descriptions and instructions that the teacher or educator can follow in order to promote, for instance, mental representations of the geometric concepts by the children.
\end{abstract}

Keywords: automata; STEM; imagery; geometry; blindness and visual impairment; inclusive education

\section{INTRODUÇÃo}

Visando a inclusão educativa de crianças cegas ou com baixa visão nas atividades do projeto Erasmus + Automata for STEM nr 2018-1-PT01-KA201-047499, tem-se vindo a desenvolver a adaptação do guião pedagógico para um dos autómatos daquele projeto, Jellybird, pretendendo-se explorar o uso daquele "brinquedo que se move", como estratégia para implementar atividades STEM através de uma pedagogía baseada no lúdico. Assim, tem-se como propósito que as crianças não só criem representações mentais do Jellybird e das partes que 0 constituem, mas também construam 0 autómato. 0 Jellybird é um pássaro, feito de papel e cartão, cujo movimento deslizar de uma das pernas provoca o bater das asas. Tendo em consideração a importância que as mensagens/informação auditivas e 0 experienciar táctil assumem na cegueira e baixa visão, e que as crianças cegas têm dificuldade em: adquirir conceitos de geometría diretamente, compreender imagens geométricas ou construir representaçoes mentais de conceitos espaciais, ao longo do processo de adaptação foram desenvolvidos novos recursos pedagógicos e apresentadas sugestões sobre como professores/as e educadores/as poderiam guiar as crianças. 0 guião pedagógico que vai ser elaborado pretende ser um complemento do guião pedagógico já existente para promover representações de conceitos geométricos. De acordo com Johnson (1987), a criança cega traz à mente as suas imagens mentais, avalia-as ou cria novas imagens. Também temos em conta as perspectivas relacionadas com o corpo na mente e cognição e a importancia de factores socioculturais para a construção do conhecimento.

0 projeto AutoSTEM visa fornecer aos/às professores/as do $1^{0}$ ciclo do Ensino Básico e educadores/as em Educação Pré-Escolar materiais para construirem um caminho didático que promova: motivação STEM; desenvolvimento de pensamento criativo, resolução de problemas e capacidade de compreensão; e consciência cultural e competências transversais (Thiel, Josephson \& Vaz-Rebelo, 2019).

Automatos são brinquedos mecânicos que poderão ser vistos como um sincretismo entre engenharia, consciência cultural e expressão artística. Os autómatos são constituídos por duas partes fundamentais: por um lado, por uma figura ou um conjunto de figuras, que podem representar uma ideia ou narrativa; por outro lado, um mecanismo que permite 0 movimento de figura(s). A abordagem pedagógica para usar autómatos pode ser baseada na combinação de narrativas e brinquedos que se movem mecanicamente, usando histórias de ciência adequadas a crianças (Thiel, Josephson \& Vaz-Rebelo, 2019).

AutoSTEM visa desenvolver uma abordagem interdisciplinar, significando que cada autómato deveria permitir às crianças, não só experimentar uma ou mais áreas STEM, mas também descobrir relações e conexões entre elas. As crianças, quando planificam e constroem um autómato, podem desenvolver diferentes competências, 
incluindo resolução de problemas, trabalho de grupo, criatividade, bem como promover a educação STEM. Por exemplo, dado que alguns autómatos representam animais, em particular o considerado neste estudo, Jellybird, representa um pássaro, assim pode ser ensinada biología (partes do corpo, o voo, como os pássaros se juntam em bandos). Ao mesmo tempo, podem ser desenvolvidos conteúdos matemáticos, tais como localização, contagem e formas geométricas (Thiel, Josephson \& Vaz-Rebelo, 2019). AutoSTEM visa também implementar uma pedagogia relacional baseada no brincar (Hedges \& Cooper, 2018), em particular uma forma de brincar guiado, considerada como uma conjugação da instrução directa e do brincar livre (Weisberg, Hirsh-Pasek, Golinkoff, Kittredge \& Klahr, 2016, p.177).

A visão é uma via fundamental para o ser humano obter informação. Quando não é possível aceder à informação através da visão, a audição e o tacto tornam-se cruciais. A audição é 0 sentido de informação e orientação da pessoa cega, permite-Ihe não só a orientação no espaço mas também no tempo. A pessoa cega ou com baixa visão percebe pela audição o mundo circundante e as pessoas, cujas vozes e sons caracterizam o espaço ambiente, o clima social ou o contexto. No entanto, não Ihe é possível registar demonstrações visuais como olhares, mímica do rosto, gestos, movimentos e equilíbrio do corpo, que são elementos fundamentais na comunicação verbal (Kohanová, 2006).

0 táctil compensa a visão no campo da comunicação gráfica (Kohanová, 2006). Mas, embora o tacto possa ser utilizado como substituto da visão para explorar o ambiente, tem limitações no alcance, distância e tamanho, de modo que a familiarização das crianças cegas com um objecto é muitas vezes incompleta. Tendem a verbalizar de forma imaginária sobre esse objeto, embora o imaginado não tenha sido experimentado ou visto directamente (Zahra, Budayasa \& Juniati, 2018).

A importância da visualização sobre a aprendizagem matemática e a imagem mental, sobre a construção de significados matemáticos, têm sido enfatizadas pelos investigadores em educação matemática. A visualização e o pensamento visual são a essência que faz da geometria um caso especial na matemática (Costa, 2005). A imagética é definida como uma colecção de imagens e o poder da imagética é que pode resultar numa visualização que ajuda as crianças a estabelecer relações e significados na aprendizagem da geometria (Solano \& Presmeg, 1995).

Zahra, Budayasa e Juniati (2018, p.2) dão enfâse à visualização, referindo que 'In elementary school, visualization becomes one of the important ability used to help students in understanding spatial concepts, shapes, sizes, and distances". A perda de experiência visual em pessoas cegas ou com baixa visão oringina dificuldades na obtenção directa dos conceitos de geometría. As crianças cegas ou com baixa visão levam muito tempo a construir uma representação mental do conceito espacial (Thinus-Blanc \& Gaunet, 1997; Vianna, Barbosa, Rocha \& Silva, 2006).

\section{OBJETIVOS DA INVESTIGAÇÃO}

0 objetivo principal da investigação consiste em adaptar o guião pedagógico e as instruções de construção do autómato Jellybird no âmbito do projeto AutoSTEM, para promover a inclusão educativa de crianças cegas ou com baixa visão nas atividades daquele projeto.

\section{PARTICIPANTES}

Os participantes integram uma equipa multidisdisciplinar em que se destacam dois técnicos em Educação Especial, sendo um deles cego, uma professora de Educação Matemática, três professores/as da área da Psicologia da Educação e estudantes da licenciatura e do mestrado em Ciências da Educação.

\section{METODOLOGIA}

A metodología de investigação adotada inspira-se no design-based research (Herrington \& Reeves, 2011) e envolve quatro fases num processo que as relaciona de acordo com a Figura 1. 
Figura 1. Processo de adaptação do guião pedagógico inspirado no design based-research de (Reeves, 2006)

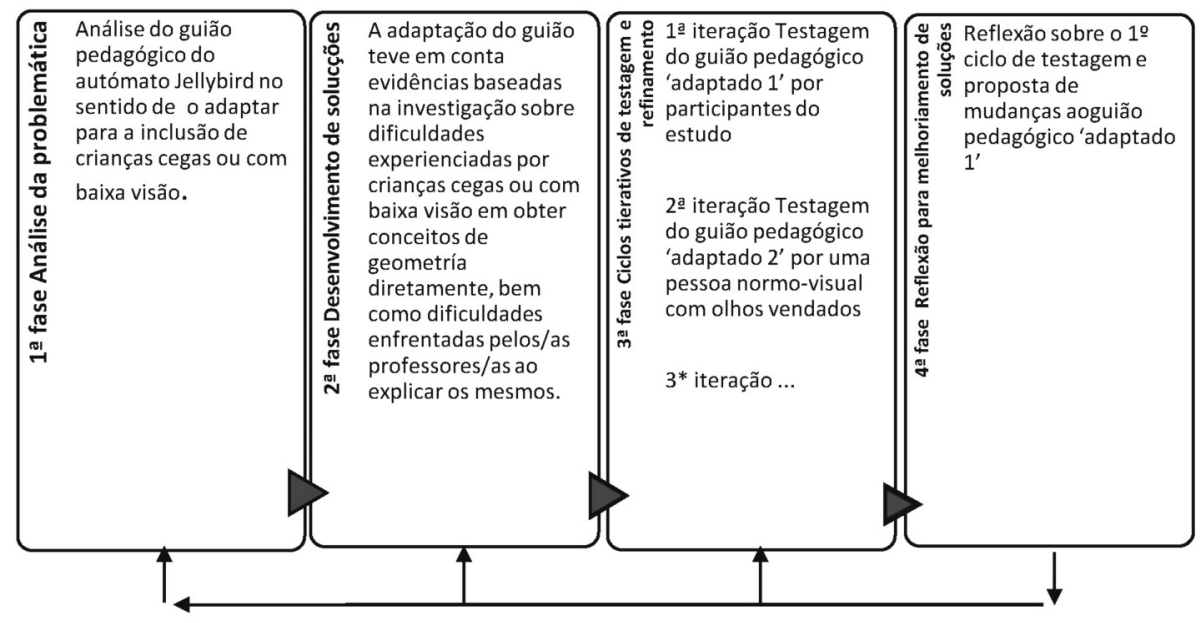

\section{$1^{\text {a }}$ fase}

0 ponto de partida foi o guião pedagógico desenvolvido no projeto AutoSTEM para o autómato Jellybird, que pode ser usado por professores/as e educadores/as, para explorar o uso do autómato com vista a promover a motivação de crianças na educação STEM. Procedeu-se também à constituição de uma equipa multidisciplinar já identificada na secção Participantes. Procedeu-se ainda à revisão de literatura sobre ensino e aprendizagem de geometria para pessoas cegas e com baixa visão.

Foi formulada a seguinte questão de investigação: Como adaptar o guião pedagógico Jellybird do projeto AutoSTEM a crianças cegas ou com baixa visão?

\section{$2^{\text {a }}$ fase}

Foram introduzidas adaptações no guião pedagógico, originando o guião pedagógico 'adaptado 1', com algumas mudanças já identificadas em Vaz-Rebelo, Costa, Bidarra, Josephson, Thiel, Santos, Gomes, Barreira, Alferes, Kostova, Bartoletti, Ferrini e Hanssen (2020). Uma das mudanças envolveu usar falas detalhadas para 0/a professor/a ou educador/a, que visam, por um lado, fazer emergir na criança as suas imagens mentais e por outro, construir novas imagens mentais. Esta imagética pretende relacionar-se com os diferentes conceitos geométricos. Também foram introduzidas tarefas que envolviam experiências auditivas, tácteis ou corpóreas; nomeadamente uso da plataforma Non-visual Desktop Access (NVDA) para a tradução de parte do guião 'adaptado 1'; figuras geométricas que constituem o Jellybird, umas representadas em relevo e outras previamente recortadas; exploração do protótipo comparando-o com o corpo humano. Foram ainda consideradas instruções de construção usando manipulativos (recortes de figuras geométricas, cordas, entre outros). Contudo estes recursos foram considerados insuficientes para a construção do autómato. Tarefas que envolvessem observação foram eliminadas.

\section{$3^{\text {a }}$ fase}

0 guião 'adaptado 1' foi testado numa sessão de cerca de duas horas, com a participação da equipa multidisciplinar. 0 material utilizado na sessão incluiu um autómato Jellybird previamente construído, uma folha A3 com as respetivas formas geométricas em relevo e também recortadas em papel. 
A sessão iniciou-se numa sala de aula com duas audições da versão do guião adaptado 1' usando a plataforma NVDA, previamente instalada num computador. De seguida, o participante cego explorou 0 autómato Jellybird previamente construído, guiado pela outra técnica de Educação Especial: identificando as diferentes partes que constituem o Jellybird, bem como o mecanismo que Ihe está associado (Figura 2); confirmando a perceção emergente quando tacteou aquelas mesmas figuras geométricas do Jellybird representadas numa folha em relevo (Figura 3); usando as figuras constituintes do Jellybird que estavam recortadas para as sobrepor com as figuras congruentes em relevo (Figura 4).

Figuras 2, 3 e 4. Ações do utilizador
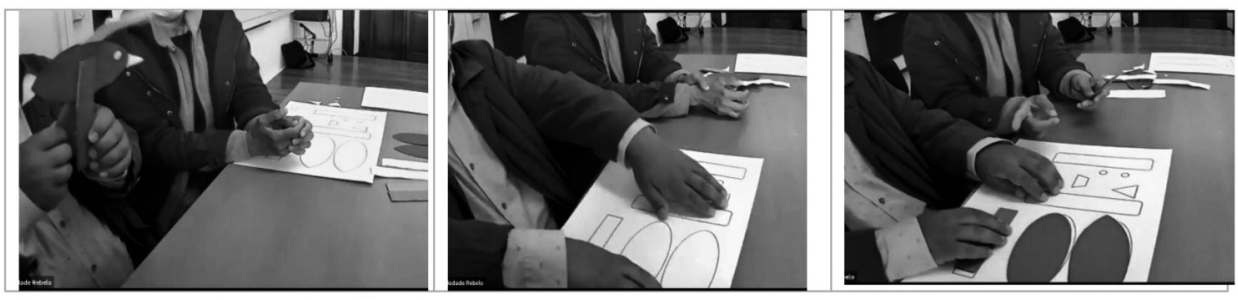

Antes de terminar a sessão o utilizador do Jellybird refletiu em grande grupo sobre a experiência que tinha vivenciado.

Durante este processo, para além de observarem, os/as outras/as participantes puderam dar sugestões, se solicitados/as, e colocar questões aos dois técnicos de Educação Especial.

\section{$4^{\text {a }}$ fase}

A sessão descrita na $3^{\underline{a}}$ fase foi analisada três dias mais tarde, numa sessão de brainstorming, num grupo de sete participantes, usando questões vindas do próprio grupo no sentido de identificar mudanças a introduzir no novo guião a adaptar'. Têm também decorrido momentos de análise dos registos vídeos das duas sessões mencionadas e identificadas mais mudanças a considerar.

\section{RESULTADOS}

A adaptação do guião pedagógico Jellybird iniciou-se com a impressão em relevo das figuras geométricas constituintes do autómato, figuras geométricas previamente recortadas e da adaptação da descrição e das instruções de construção e sua apresentação oral através da plataforma NVDA.

Após $01^{0}$ ciclo iterativo e a reflexão associada, foi possível identificar áreas de melhoria no guião pedagógico, sendo propostas as seguintes alterações adicionais:

Apresentar inicialmente uma síntese da tarefa a realizar.

Simplificar a descrição do autómato. Esta descrição poderá incluir uma breve referència às partes constituintes do autómato e ao seu movimento.

A informação deve ser apresentada em frases curtas.

Coordenar a informação audio com o tempo de exploração táctil.

Dar ênfase à exploração pela criança do autómato, apoiado por questionamento guidado.

As descrições do guião poderão ser sugestões de falas para o professor ou educador usar em função da especificidade do contexto onde as atividades decorrem.

Adaptar o protótipo JellyBird de modo a criança o poder desmontar e reconstruir.

\section{DISCUSSÃO}

Partindo das dificultades de crianças cegas ou com baixa visão na compreensão e representação mental de 
conceitos de geometria, as adaptações introduzidas no guião pedagógico procuram ir ao encontro de resultados de investigação (Costa, 2005, Kohanová, 2006, Solano \& Presmeg, 1995; Thinus-Blanc \& Gaunet, 1997; Vianna, Barbosa, Rocha \& Silva, 2006, Zahra, Budayasa \& Juniati, 2018) que apontam para a importância de percursos pedagógicos que envolvam experiências do dia a dia, corpóreas, auditivas e tácteis, visando a construção de uma imagética visual de forma a compreender conceitos geométricos.

Estes percursos pedagógicos podem ser mediados por recursos digitais e humanos que se interligam de forma multimodal e que introduzem desafíos para fomentar uma educação STEM e envolver profesores/as do $1^{0}$ ciclo do Ensino Básico e educadores/as.

\section{CONCLUSÕES}

A adaptação do guião pedagógico, visando a inclusão de crianças cegas ou com baixa visão nas atividades do projeto AutoSTEM, está a ser desenvolvida e poderá emergir de vários ciclos de testagem iterativos envolvendo diferentes agentes, contextos e perspetivas de investigação.

A introdução da atividade de 'desmontar' o autómato' no guião pedagógico parece ser uma janela para fomentar a compreensão de conceitos geométricos e espaciais e permitir à criança cega ou com baixa visão construir o Jellybird, individualmente ou em cooperação com os seus pares normovisuais, vivenciando assim uma educação STEM, a par com outras crianças.

\section{REFERÊNCIAS BIBLIOGRÁFICAS}

Costa, C. (2005). A model for visual-spatial thinking: Geometric transformations in early scholarity. Dissertation submitted to New University of Lisbon, Portugal for the degree of Doctor of Science of Education.

Hedges, H. \& Cooper, M. (2018). Relational play-based pedagogy: Theorising a core practice in early childhood education. Teachers and Teaching, 24(4), 369-383.

Herrington, J. \& Reeves, T.C. (2011). Using design principles to improve pedagogical practice and promote student engagement. In G. Williams, P. Statham, N. Brown \& B. Cleland (Eds.), Changing Demands, Changing Directions. Proceedings ascilite Hobart 2011. (pp.594-601).

Johnson, M. (1987). The body in the mind: The bodily basis of meaning, imagination and reason. The University of Chicago Press.

Kohanová, I. (2006). Teaching mathematics to non-sighted students: With specialization in solid geometry. Dissertation submitted to Comenius University of Bratislava for the degree of Doctor.

Pritchard, C. K. \& Lamb (2012), Teaching geometry to visually impaired students. Journal of Mathematics Teacher, 106, 22-27

Reeves, T. C. (2006). Design research from a technology perspective. In J. van den Akker, K. Gravemeijer, S. McKenney \& N. Nieveen (Eds.), Educational design research (pp. 52-66). Routledge.

Solano, A. \& Presmeg, N. (1995). Visualization as a relation of images. Proceedings of the 19 th International Conference for the Psychology of Mathematics Education (Vol 3, pp. 66-73). Brasil: University of Pernambuco

Thiel, 0., Josephson, J., \& Vaz-Rebelo, P. (2019). Automata for STEM: Step by step teacher guide. Retrieved from https://www.autostem.info/wp-content/uploads/2019/12/AutoSTEM-Teacher-guide.pdf

Thinus-Blanc, C. \& Gaunet, F. (1997). Space representations in the blind: Vision as a spatial sense? Psychological Bulletin 121, 20-42

Vaz-Rebelo, P., Costa, C., Bidarra, G., Josephson, J., Thiel, O., Santos, A., Gomes, R., Barreira, C., Alferes, V., Kostova, N., Bartoletti, C., Ferrini, F., Hanssen, S., (2020) Instructions to promote mental representation of geometric shapes in children with visual impairment when constructing a moving toy: an example from AutoSTEM project. ICERI2020 Proceedings, pp.9835-9839. http://dx.doi.org/10.21125/iceri.2020.2204

Vianna, C.S., Barbosa, P. M., Rocha, D.F \& B. Silva, B. (2006). Teaching geometry for blind and visually impaired students. International Congress on Mathematical Education. 
Zahra, A., Budayasa, I. K., \& Juniati, D. (2018), The blind student's interpretation of two-dimensional shapes in geometry. Journal of Physics: Conference Series 947, 1-6.

Zahra, A., Juniati, D. \& Budayasa, I. K. (2018), A study of geometry concept mathematization process on blind student visual imagery. International Journal of Engineering \& Technology, v. 7, n. 4.30, p. 89-93, Available at: https://www.sciencepubco.com/index.php/ijet/article/view/22023>. Date accessed: 24

Weisberg, D., Hirsh-Pasek, K., Golinkoff, R, M., Kittredge, A. K. \& Klahr, D. (2016). Guided Play: Principles and Practices. Current Directions in Psychological Science, 25(3), 177-182. DOI: 10.1177/0963721416645512. 
